\title{
Synchronous Adie's syndrome and type 1 antineuronal nuclear antibody (anti-Hu)-related paraneoplastic neurological syndromes as predictors of complete response in limited-stage small-cell lung cancer: A case report
}

\author{
KATHERINA BERNADETTE SRETER ${ }^{1}$, BLAZENKA BARISIC ${ }^{2}$, \\ MARIJA BARISIC KUTIJA ${ }^{3}$, SUZANA KUKULJ ${ }^{4-6}$ and MIROSLAV SAMARZIJA ${ }^{5,7}$
}

\begin{abstract}
${ }^{1}$ Department of Clinical Immunology, Pulmonology and Rheumatology, University Hospital Centre 'Sestre Milosrdnice';
${ }^{2}$ Department of Allergy and Chronic Obstructive Pulmonary Disease, Clinic for Respiratory Diseases 'Jordanovac', University Hospital Centre Zagreb; ${ }^{3}$ Department of Ophthalmology, University Hospital Centre Zagreb; ${ }^{4}$ Department of Mediastinal Tumours, Clinic for Respiratory Diseases 'Jordanovac', University Hospital Centre Zagreb; ${ }^{5}$ School of Medicine, University of Zagreb, 10000 Zagreb, Croatia; ${ }^{6}$ School of Medicine, University of Mostar, 88000 Mostar, Bosnia and Herzegovina;

${ }^{7}$ Department of Post-Intensive Care, Clinic for Respiratory Diseases 'Jordanovac', University Hospital Centre Zagreb, 10000 Zagreb, Croatia
\end{abstract}

Received November 9, 2016; Accepted March 17, 2017

DOI: $10.3892 / \mathrm{mco} .2017 .1248$

\begin{abstract}
Adie's syndrome (AS) and paraneoplastic sensorimotor neuropathy with cerebellar ataxia (PSN CA) are extremely rare, rapidly progressive, autoimmune diseases associated with the development of antibodies against neuronal-specific $\mathrm{Hu}$ proteins that are abnormally expressed in small-cell lung cancer (SCLC). We herein present the unique case of a 55-year-old obese woman, previous heavy smoker, who, during treatment with standard cisplatin-etoposide chemotherapy for limited-stage SCLC, developed simultaneous AS and worsening symptoms consistent with PSN CA that led to significant neurological disability and severe axonal electrophysiological pattern on nerve conduction studies. Serology confirmed the presence of low-titre type 1 antineuronal nuclear antibodies (ANNA-1), previously referred to as anti-Hu antibodies. Following plasmapheresis, immunosuppressive therapy and physical rehabilitation, the neurological symptoms progressively improved. The tumour completely regressed, with no recurrence detected on subsequent radiological examinations. The aim of this case was to highlight the importance of a multidisciplinary team approach for early recognition and rapid treatment of paraneoplastic neurological syndromes (PNS) as
\end{abstract}

Correspondence to: Dr Katherina Bernadette Sreter, Department of Clinical Immunology, Pulmonology and Rheumatology, University Hospital Centre 'Sestre Milosrdnice', 29 Vinogradska, 10000 Zagreb, Croatia

E-mail: ksreter@yahoo.com

Key words: Adie's syndrome, anti-Hu antibodies, paraneoplastic syndromes, polyneuropathies, small-cell lung carcinoma key to achieving significant recovery and marked improvement of the neurological deficit. This report extends the literature by confirming earlier studies showing that the presence of serum ANNA-1 in SCLC, an aggressive type of pulmonary carcinoma that is challenging to treat, may portend a more favourable prognosis and response to chemotherapy. Thus, patients with SCLC and new-onset neurological symptoms should be tested for ANNA-1. The role of a multimodality approach to treating PNS is also emphasized.

\section{Introduction}

Small-cell lung cancer (SCLC), the most aggressive subtype of pulmonary carcinoma, accounts for up to $15 \%$ of all newly diagnosed lung cancers, with an overall 5-year survival rate of only $6 \%(1,2)$. The initial response to chemotherapy may be favourable, but relapse is common (3). Paraneoplastic neurological syndromes (PNS) represent a wide spectrum of rare autoimmune diseases that may often be the first manifestation of an underlying SCLC (4). Among cancer patients, $<1 \%$ overall develop PNS, but it occurs in up to $3-5 \%$ of those with SCLC (5). These severe, often rapidly progressive and debilitating neuropathies are frequently associated with type 1 antineuronal nuclear antibodies (ANNA-1), previously referred to as anti-Hu antibodies (6).

The $\mathrm{Hu}$ antigens are intracellular proteins, normally expressed throughout the central nervous system (CNS) and peripheral nervous system (7). In healthy adults, anti-Hu antibodies are not detected in the serum, as the blood-brain barrier sequesters the developing CNS from the immune system (5). Interestingly, all SCLCs also express the $\mathrm{Hu}$ antigen (specifically $\mathrm{HuD}$ ) (8). Detectable levels of circulating anti-Hu antibodies may be found in $20 \%$ of SCLC patients, although not all will develop PNS $(5,9)$. It remains 
unclear why only some SCLC patients develop PNS (10). It has been hypothesized that these ectopic neuronal antigens are recognized by the immune system as foreign, triggering the production of ANNA-1 with consequential paraneoplastic effects manifesting clinically with various degrees of severity and neurological symptoms $(7,8)$.

When detected in the serum or cerebrospinal fluid (CSF), anti-Hu antibodies have been associated with a wide spectrum of neurological and neuro-ophthalmological disorders presenting as PNS (11). Adie's syndrome (AS), namely tonic pupil with areflexia, has been previously reported as a rare neuro-ophthalmic presentation of anti-Hu paraneoplastic syndrome associated with SCLC (11). It is hypothesized that concurrent loss of parasympathetic ciliary ganglia and dorsal root ganglionic cells may be responsible for AS $(12,13)$. We herein present a unique case of concomitant AS and rapidly progressive PNS associated with ANNA-1, with complete response of chemotherapy-treated SCLC and subsequent partial recovery of the neurological deficit following multimodal therapy, with long-term stability.

\section{Case report}

In December 2012, a 55-year-old woman, former heavy smoker (53 pack-years), was admitted to the Clinic for Respiratory Diseases 'Jordanovac', University Hospital Centre Zagreb (Zagreb, Croatia) due to hemoptysis and evaluation of a pulmonary nodule in the right lung field juxtaposed to the right hilum on chest X-ray (Fig. 1A). The patient's past medical history included hypercholesterolemia, for which she was being treated with simvastatin. A computed tomography (CT) scan of the thorax revealed a proliferative nodule $(1.7 \mathrm{~cm}$ in diameter) in the lateral basal bronchopulmonary segment of the right lower lobe. Following fiberbronchoscopy, the clinical stage was determined to be IIIA (T1aN2M0) or limited-stage (localised disease) SCLC. A chemotherapy regimen with cisplatin and etoposide was immediately initiated. By the third cycle of chemotherapy (February 2013), the patient began to experience paresthesia and weakness of the right hand. The head CT and magnetic resonance imaging (MRI) scans were unremarkable. Physical examination detected anisocoria. The patient was promptly referred to the staff ophthalmologist and neurologist.

Ocular examination revealed a right mydriatic pupil, without direct or consensual pupillary constriction to light, and no constriction on attempted accommodation. A neuro-ophthalmologist was consulted. Following local instillation of one drop of diluted pilocarpine $(0.1 \%)$ in each eye, the affected pupil showed cholinergic supersensitivity by constricting, a finding consistent with unilateral Adie's pupil. The nerve conduction study pointed to a suspected peripheral neuropathy congruent with the presenting clinical characteristics. The etiology was hypothesized to be associated with the underlying malignancy. The treating neurologist recommended immunological work-up, particularly serological tests for antinuclear antibodies, to evaluate the possibility of a PNS. Autoantibody screening was positive for anti-Hu antibody at a low titre.

By the sixth cycle of chemotherapy, the patient was exhibiting symptoms of further peripheral sensory and motor neurological decline. A repeat brain MRI scan was again unremarkable. Follow-up electromyoneurography indicated profound neurological and electromyographic progression. Nerve conduction studies showed severe axonal sensorimotor polyneuropathy with sensory predominance, and signs of cerebrospinal toxic-like lesions. Based on the results of the electrophysiological study, neurological examination and earlier positive serum ANNA-1, it was concluded that the patient was suffering from a PNS, more specifically peripheral neuropathy with cerebellar symptomatology. A planned positron emission tomography-CT scan and radiotherapy were not performed due to the patient's poor performance status.

Within 2 months, the patient was bedridden, no longer able to tolerate sitting due to vertigo, and experiencing uncontrolled movements of her right hand and head tremor; her speech was laboured with discrete dyslalia and she exhibited horizontal nystagmus on left and right gaze. The muscle tone was decreased overall, most prominently in the right extremities. Areflexia of both the upper and lower extremities was observed. Due to worsening of her clinical condition, the patient underwent plasmapheresis treatments (five in total) through a central venous catheter every second day. Each treatment consisted of 3,500 $\mathrm{ml}$ of intravenous $5 \%$ albumin and concomitant oral methylprednisolone at $32 \mathrm{mg}$. The patient tolerated the therapy well and exhibited a minor improvement of the neurological status. A higher dose of oral methylprednisolone (48 mg daily) was recommended at discharge.

By September 2013, the patient's condition had declined further. Given that the plasmapheresis treatments had not led to any significant improvement in neurological status, cyclophosphamide at one 50-mg tablet daily was added to her treatment, along with valproate and clonazepam. The myoclonus improved during in-hospital treatment. The patient was re-evaluated in March and October 2014. Further neurological recovery was observed. The motor coordination of her right hand improved and she was able to independently sit and walk a few steps with assistance. In addition to the medication, continuous and intense physical rehabilitation at home and in-hospital proved key to the patient's improvement. She continued to exhibit a slow but steady recovery of neurological functioning over the next year. In November 2014, serum ANNA-1 remained positive at a low titre. At the clinical visit (late November 2015), the patient's neurological status and overall health were stable. The latest telephone update 1 year later (early November 2016) was promising, as the patient reported her condition to be stable.

Partial tumour regression was observed on chest X-ray after the first cycle of chemotherapy. Subsequent follow-up radiological examinations remained unchanged, until complete response was noted 6 months later in May 2013 (Fig. 1B), as well as on subsequent visits. The latest follow-up radiological examinations [thoracic CT scan in May 2015 and chest X-ray in November 2015 (Fig. 1C)] revealed no tumour recurrence.

\section{Discussion}

Anti-Hu antibodies are a polyclonal IgG type of antibody that bind to intracellular antigens found in neural as well as tumour cells, thus leading to immune cross-reactivity $(9,14)$. This simultaneous attack of neurons and malignant cells may cause PNS symptoms and slow down tumour growth (14). In $88 \%$ of the cases, the presence of anti-Hu antibodies is associated 

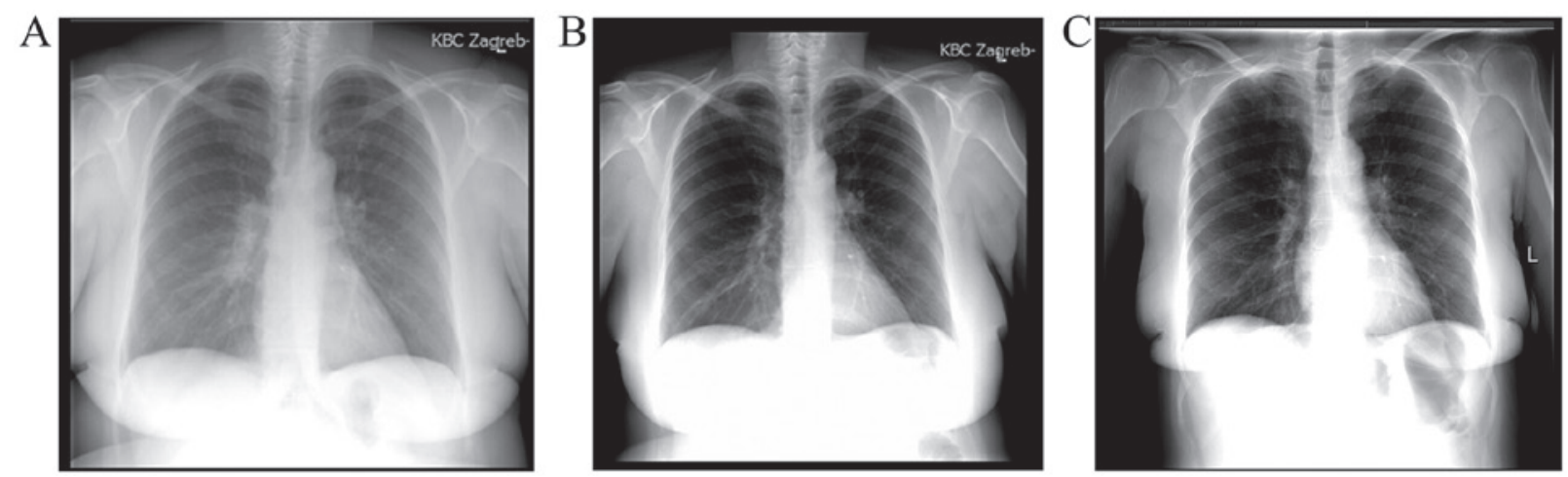

Figure 1. Chest X-rays (A) at first presentation in November 2012, revealing an infiltrating proliferative nodule in the right lung field juxtaposed to the right hilum, (B) at the 6-month follow-up, showing complete tumour regression and (C) at the latest follow-up in November 2015, without signs of tumour recurrence.

with an underlying neoplasm; of those, 81\% are SCLCs (15). SCLC is reported to account for $>90 \%$ of cases of PNS related to ANNA-1 (5). The present case highlights the importance of considering rare associations as possible diagnoses.

Chemotherapy is generally not considered to be effective in SCLC, as recurrence is inevitable in the majority of patients, regardless of the extent of the disease $(3,16)$. However, the presence of ANNA-1 appears to be protective (17), and has been shown in several reports to be associated with increased tumour chemosensitivity, indolent tumour growth, complete response to therapy, and prolonged survival $(6,15,16,18-22)$. Spontaneous tumour regression associated with ANNA-1 in patients with histologically proven SCLC, without any active treatment, has also been described in 2 cases in the literature $(7,23)$, and in 1 rare case of non-SCLC (24). The present study adds further evidence to support the theory that ANNA-1 in the serum may confer antitumour activity, causing regression of lung neoplasms (7).

In the case presented herein, unilateral Adie's pupil and peripheral sensorimotor neuropathy with cerebellar symptomatology developed during chemotherapy for limited-stage SCLC. Anti-Hu antibodies were detected in the patient's serum at a low titre, which is a highly uncommon finding, as the patient also had Hashimoto's thyroiditis. Low antibody titres are usually present in SCLC patients without autoimmune diseases (25). Those with SCLC and no clinical PNS have detectable anti-Hu antibodies in their serum in $\sim 16-25 \%$ of the cases (25-27). When SCLC and PNS are found concurrently, the latter is usually mediated by a high titre of anti-Hu antibodies (28).

According to the limited number of cases reported in the literature, a multimodality approach to the treatment of PNS, consisting of immunosuppressive therapy with a combination of intravenous immunoglobulin, methylprednisolone and cyclophosphamide, may transiently stabilize the disorder (5). However, to the best of our knowledge, there are currently no studies reporting long-term improvement in PNS (5). It appears that a vigorous immunosuppressive treatment combining high doses of steroids, cyclophosphamide and intravenous immunoglobulins in anti-Hu-associated PNS (regardless of the type) in severely disabled patients at the onset of treatment is not beneficial (29). It has been reported that $65 \%$ of patients with SCLC and paraneoplastic encephalomyelitis with detectable serum ANNA-1 succumb to neurological complications rather than tumour progression $(6,16)$. The prognosis is poor, with the median survival for anti-Hu patients following the onset of treatment reported to be 6 months (range, 3-35 months) (29). The recent discovery that ANNA-1 may cause neuronal cell death may account for the irreversible nature of paraneoplastic neurological deficits in patients with these autoantibodies (30).

Although the exact underlying pathological mechanism has not been fully elucidated, it is likely that humoral immunity may not be solely responsible for the incidence of PNS. Several types of T cells may in fact be present in different PNS patients, and these variants may explain the spectrum of clinical outcomes that have been described in the literature thus far with regards to tumour response and PNS severity (31). A number of experimental studies have suggested that cytotoxic T cells may be the main effectors of the immune response (32). This T-cell mediated response may result in neuronal cell death and axonal degeneration that are extremely difficult to treat $(32,33)$. It remains unclear, however, why only some SCLC patients develop PNS, given that the HuD antigen is constantly expressed by these tumour cells (29).

A few exceptional reports of neurological recovery have been described in the literature, both spontaneous and following immunosuppressor treatment alone (29). In the present case, the patient was already bedridden at the time of initiation of multimodal immunosuppressive therapy for anti-Hu-associated PNS. The positive outcome described herein may be attributed to the addition of plasmapheresis to the treatment regimen, which may have exerted a synergistic effect. Interestingly, however, it has been demonstrated that plasmapheresis reduces anti-Hu antibody levels in the serum, but not in the CSF (10). Unlike the majority of previous reports of imminent death from progressive PNS, the patient described herein has achieved steady recovery of the neurological status over the course of the past 45 months since the first presentation of neurological deficit, and has remained stable.

In general, PNS should be highly suspected in a SCLC patient who develops a progressive neurological and/or neuro-ophthalmic disorder in a subacute course after all other possible causes have been excluded (5). The presence of anti-neural antibodies, such as anti-Hu, should be investigated in the patient's serum. A multidisciplinary team approach cannot be underestimated, as it entails a comprehensive investigation of the presenting symptoms. We recommend that both a neurologist and ophthalmologist be included in the 
initial diagnostic process and subsequent regular follow-ups. Similarly, in patients with neurological symptoms, but without known lung cancer diagnosis, a search for anti-Hu may reveal an underlying tumour, such as SCLC. Given that there is currently no screening tool for early SCLC detection, ANNA-1 and other SCLC-related autoantibodies may prove useful as antibody-based early cancer detection biomarkers. Discovering SCLC when the malignancy is at an early stage and undetectable by available radiological methods should lead to better prognosis and prolong survival. Further research using animal-based models to study immunological events that occur during SCLC growth may provide insight into the process that drives the development of paraneoplastic disease in humans. Translating these results into clinical practice may enable initiating treatment during a window of better functional state, leading to improved clinical outcomes.

Written informed consent was obtained from the patient for publication of this manuscript and any accompanying radiological images.

\section{References}

1. Howlader N, Noone AM, Krapcho M, Miller D, Bishop K, Altekruse SF, Kosary CL, Yu M, Ruhl J, Tatalovich Z, et al. (eds): SEER Cancer Statistics Review, 1975-2013, November 2015, National Cancer Institute. Bethesda, MD, http://seer.cancer. gov/csr/1975 2013/, based on SEER data submission, posted to the SEER web site. Accessed April 25, 2016.

2. Worden FP and Kalemkerian GP: Therapeutic advances in small cell lung cancer. Expert Opin Investig Drugs 9: 565-579, 2000.

3. Kalemkerian GP: Advances in pharmacotherapy of small cell lung cancer. Expert Opin Pharmacother 15: 2385-2396, 2014

4. Darnell RB and Posner JB: Paraneoplastic syndromes involving the nervous system. N Engl J Med 349: 1543-1554, 2003.

5. Kanaji N, Watanabe N, Kita N, Bandoh S, Tadokoro A, Ishii T, Dobashi $\mathrm{H}$ and Matsunaga T: Paraneoplastic syndromes associated with lung cancer. World J Clin Oncol 5: 197-223, 2014.

6. Dalmau J, Graus F, Rosenblum MK and Posner JB Anti-Hu-associated paraneoplastic encephalomyelitis/sensory neuronopathy. A clinical study of 71 patients. Medicine (Baltimore) 71: 59-72, 1992.

7. Mawhinney E, Gray OM, McVerry F and McDonnell GV: Paraneoplastic sensorimotor neuropathy associated with regression of small cell lung carcinoma. BMJ Case Reports 2010: pii: bcr0120091486, 2010.

8. Roberts WK, Deluca IJ, Thomas A, Fak J, Williams T, Buckley N, Dousmanis AG, Posner JB and Darnell RB: Patients with lung cancer and paraneoplastic $\mathrm{Hu}$ syndrome harbour HuD-specific type 2 CD8+ T cells. J Clin Invest 119: 2042-2051, 2009.

9. Gozzard P and Maddison P: Which antibody and which cancer in which paraneoplastic syndromes? Pract Neurol 10: 260-270, 2010.

10. Pignolet BS, Gebauer CM and Liblau RS: Immunopathogenesis of paraneoplastic neurological syndromes associated with anti-Hu antibodies: A beneficial antitumor immune response going awry. Oncoimmunology 2: e27384, 2013.

11. Bruno MK, Winterkorn JM, Edgar MA, Kamal A and Stübgen JP: Unilateral Adie pupil as sole ophthalmic sign of anti-Hu paraneoplastic syndrome. J Neuroophthalmol 20: 248-249, 2000.

12. Harriman DG and Garland H: The pathology of Adie's syndrome. Brain 91: 401-418, 1968.

13. Hersh B, Dalmau J, Dangond F, Gultekin S, Geller E and Wen PY: Paraneoplastic opsoclonus-myoclonus associated with anti-Hu antibody. Neurology 44: 1754-1755, 1994.

14. Yin L, Qu H and Chen Q: Proliferative response of peripheral blood mononuclear cells in anti-Hu antibody-associated patients with paraneoplastic neurological syndrome and their depressant effect on small cell lung cancer cells. Mol Med Rep 11: 1595-1600, 2015.
15. Graus F, Dalmou J, Reñé R, Tora M, Malats N, Verschuuren JJ, Cardenal F, Viñolas N, Garcia del Muro J, Vadell C, et al: Anti-Hu antibodies in patients with small-cell lung cancer: Association with complete response to therapy and improved survival. J Clin Oncol 15: 2866-2872, 1997.

16. Douglas CA and Ellershaw J: Anti-Hu antibodies may indicate a positive response to chemotherapy in paraneoplastic syndrome secondary to small cell lung cancer. Palliat Med 17: 638-639, 2003.

17. Kazarian M, Calbo J, Proost N, Carpenter CL, Berns A and Laird-Offringa IA: Immune response in lung cancer mouse model mimics human anti-Hu reactivity. J Neuroimmunol 217: 38-45, 2009.

18. Dalmau J, Graus F, Cheung NK, Rosenblum MK, Ho A, Cañete A, Delattre JY, Thompson SJ and Posner JB: Major histocompatibility proteins, anti-Hu antibodies and paraneoplastic encephalomyelitis in neuroblastoma and small cell lung cancer. Cancer 75: 99-109, 1995.

19. Sillevis Smitt P, Grefkens J, de Leeuw B, van den Bent M, van Putten W, Hooijkaas H and Vecht C: Survival and outcome in 73 anti-Hu positive patients with paraneoplastic encephalomyelitis/sensory neuronopathy. J Neurol 249: 745-753, 2002.

20. Aguirre-Cruz L, Charuel JL, Carpentier AF, Benyahia B, Delattre JY and Musset L: Clinical relevance of non-neuronal auto-antibodies in patients with anti-Hu or anti-Yo paraneoplastic diseases. J Neurooncol 71: 39-41, 2005.

21. Maddison P and Lang B: Paraneoplastic neurological autoimmunity and survival in small-cell lung cancer. J Neuroimmunol 201-202: 159-162, 2008.

22. Tsou JA, Kazarian M, Patel A, Galler JS, Laird-Offringa IA, Carpenter CL and London SJ: Low level anti-Hu reactivity: A risk marker for small cell lung cancer? Cancer Detect Prev 32: 292-299, 2009

23. Gill S, Murray N, Dalmau J and Thiessen B: Paraneoplastic sensory neuronopathy and spontaneous regression of small cell lung cancer. Can J Neurol Sci 30: 269-271, 2003.

24. Pujol JL, Godard AL, Jacot W and Labauge P: Spontaneous complete remission of a non-small cell lung cancer associated with anti-Hu antibody syndrome. J Thorac Oncol 2: 168-170, 2007.

25. Kazarian M and Laird-Offringa IA: Small-cell lung cancer-associated autoantibodies: Potential applications to cancer diagnosis, early detection, and therapy. Mol Cancer 10: 33, 2011.

26. Voltz RD, Posner JB, Dalmau J and Graus F: Paraneoplastic encephalomyelitis: An update of the effects of the anti-Hu immune response on the nervous system and tumour. J Neurol Neurosurg Psychiatry 63: 133-136, 1997.

27. Graus F, Keime-Guibert F, Reñe R, Benyahia B, Ribalta T, Ascaso C, Escaramis G and Delattre JY: Anti-Hu-associated paraneoplastic encephalomyelitis: Analysis of 200 patients. Brain 124: 1138-1148, 2001.

28. List M, Jamous F, Gupta A and Huntington M: Anti-Hu positive antibodies and small cell carcinoma: A single center review. S D Med 68: 251-255, 2015.

29. Keime-Guibert F, Graus F, Fleury A, René R, Honnorat J, Broet P and Delattre JA: Treatment of paraneoplastic neurological syndromes with antineuronal antibodies (Anti-Hu, Anti-Yo) with a combination of immunoglobulins, cyclophosphamide and methylprednisolone. J Neurol Neurosurg Psychiatry 68: 479-482, 2000

30. Greenlee JE, Clawson SA, Hill KE, Wood B, Clardy SL, Tsunoda I, Jaskowski TD and Carlson NG: Neuronal uptake of anti-Hu antibody, but not anti-Ri antibody, leads to cell death in brain slice cultures. J Neuroinflammation 11: 160, 2014.

31. Roberts WK, Deluca IJ, Thomas A, Fak J, Williams T, Buckley N, Dousmanis AG, Posner JB and Darnell RB: Patients with lung cancer and paraneoplastic $\mathrm{Hu}$ syndrome harbor HuD-specific type 2 CD8+T cells. J Clin Invest 119: 2042-2051, 2009.

32. Antoine JC: Peripheral neuropathies associated with antibodies directed to intracellular neural antigens. Rev Neurol (Paris) 170: 570-576, 2014

33. Pignolet BS, Gebauer CM and Liblau RS: Immunopathogenesis of paraneoplastic neurological syndromes associated with anti-Hu antibodies: A beneficial antitumor immune response going awry. Oncoimmunology 2: e27384, 2013. 